\title{
Nanostructured lipid carrier delivering chlorins e6 as in situ dendritic cell vaccine for immunotherapy of gastric cancer
}

\author{
Mao Mao ${ }^{\text {b) }}$ Senfeng Lilu', Yiming Zhou, Gonghe Wang, Jianping Deng, Lei Tian'a) \\ Department of Gastric Gland Surgery, First Affiliated Hospital of Guangxi Medical University, Nanning, Guangxi 530021, China

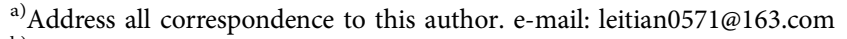 \\ b) These authors contributed equally to this work. \\ Received: 7 May 2020; accepted: 3 August 2020
}

The recent scientific progress has shown the promising effect of the vaccine in immunotherapy of cancer, which relies on the antigen processing/presentation capability of dendritic cells (DCs). As a result, cancer vaccines targeting DC, which also named as DC vaccine, was a hot-spot in vaccine development. Herein, a nanostructured lipid carrier (NLC) was employed to load chlorin e6 (Ce6) to serve as a potential in situ DC vaccine (NLC/Ce6) for effective immunotherapy of gastric cancer. Taking advantage of the photodynamic effect of Ce6 to generate reactive oxygen species (ROS) under laser irradiation, the $\mathrm{NLC} / \mathrm{Ce} 6$ was able to trigger cell death and expose tumor-associated antigen (TAA). Moreover, mimicking the natural inflammatory response, the ROS can also recruit the DC for the effective processing/presentation of the in situ exposed TAA. As expected, we observed strong capability DC vaccination efficacy of this platform to effectively inhibit the growth of both primary and distant gastric tumors.

\section{Introduction}

Cancer immunotherapy is becoming one of the most promising means in cancer management. In particular, the dendritic cells (DCs)-based cancer vaccine showed irreplaceable advantages over other counterparts, including low cost and high safety $[1,2]$. As a result, the DC vaccine is becoming a hot-spot of in cancer therapy $[3,4]$. Considering the critical role of DCs in the activation of immune responses, the effective antigen processing/presentation of DC is the major obstacle in the development of the DC vaccine [5]. Nonetheless, current DC vaccines usually require complicated processing of related products and the reproducibility is poor and the cost is relatively high [6]. As a result, finding an easy and repeatable approach to trigger effective DC activation for the immune response might be challenging, but is the future of the DC vaccine [7].

The introduction of nanotechnology is a great leap for the advance of vaccine development $[8,9,10,11]$. However, previous reported nanotechnology-based vaccines usually require repeated injections and relatively high doses to afford the barely feasible immune response $[12,13,14]$. Therefore, the development of a formulation capable of inducing a strong immune response with reduced dosage is greatly needed. Nanostructured lipid carrier (NLC) as one of the most wellrecognized carriers in drug delivery has shown many virtues over other counterparts, including decent drug loading, preferable biocompatibility, as well as easy fabrication. As a result, many NLC-based drug delivery systems (DDSs) have been proposed for cancer therapy with satisfying performance $[15,16]$. Using the enhanced permeability and retention (EPR) effect in tumor sites and the overexpressed antigens on the cancer cell surface, the tumor-targeted drug delivery is becoming more and more assessable $[16,17]$. However, the NLC-based vaccines for cancer immunotherapy have rarely been explored, which might be an interesting topic.

Photodynamic therapy (PDT) has been shown by many previous reports to inhibit the growth of tumors through the killing effect of reactive oxygen species (ROS). Recent studies have also revealed the expose of tumor-associated antigen (TAA) in PDT treated tumor cells $[18,19]$. Considering that naturally occurred inflammation also involves the elevation of the ROS level, we therefore suggested that the ROS in PDT might be able to mimic the naturally inflammatory response 
to induce the activation of the immune response [20, 21]. By using a well-designed DDS for PDT of tumor, the exposed TAA was suggested to activate the following DC to afford in situ vaccination. This is a more effective way than the traditional DC vaccine and might be able to reduce the dosage and cycle with acceptable immunotherapy outcome [22, 23].

In our research, we developed a folic acid (FA)-modified NLC vehicle for the loading of PDT agent chlorin e6 (NLC/ Ce6). The as-prepared DDS was capable of serving as a potential in situ DC vaccine for effective immunotherapy of gastric cancer. The NLC is capable of loading Ce6 through hydrophobic interactions. The PDT effect of Ce6 was expected to induce structure damage of tumor tissue and apoptosis of cancer cells to better expose and spread TAA. In particular, the ROS generated by PDT was expected to effectively recruit (inflammation mimic) DC cells to realize in situ cancer vaccination.

\section{Results and Discussions}

\section{Preparation and characterization of $\mathrm{NLC} / \mathrm{Ce} 6$}

The NLC/Ce6 was prepared in a one-pot method using the solvent-diffusion method. The FA was modified to the surface of NLC during the preparation process.

As displayed in Fig. 1(a), the prepared NLC/Ce6 is uniformly dispersed nanoparticles with a narrow size distribution around $100 \mathrm{~nm}$. Previous reports reported that compared with other size ranges for targeted drug delivery, nanoparticles with a size of approximately $100 \mathrm{~nm}$ are most suitable for taking advantage of the tumor-specific EPR effect [24, 25, 26].

To test the long-term stability of NLC/Ce6, we cultured $\mathrm{NLC} / \mathrm{Ce} 6$ in PBS and plasma for $48 \mathrm{~h}$ and monitored the change of particle size during this period to determine its stability. As displayed in Fig. 1(b), throughout the cycle, the diameter of NLC/Ce6 only shows small fluctuations (less than $10 \%$ ), which indicates that the stability of NLC/Ce6 can be well maintained under physiological conditions, so it is very suitable to be used as DDS in cancer treatment.

To further verify the safety of NLC/Ce6, we also conducted a hemolysis test. As shown in Fig. 2(a), after incubating in 2\%
RCB for $1 \mathrm{~h}$, it was observed that the hemolysis rate of NLC/ Ce6 was positively correlated with the DDS concentration. However, the hemolysis rate was less than $1.2 \%$ at all tested concentrations. Considering that the plasma concentration of nanoparticles in practical applications will be much lower than the concentration used in the experiment, we speculate that NLC/Ce6 has no risk of hemolysis.

Although NLC/Ce6 can provide well protection for the encapsulated drug, if the packaged Ce6 can still activatable upon laser stimulation to show its photodynamic effect is to be studied. Therefore, we used DPBF (diphenylisobenzofuran) as a ROS detector in our experiments to determine the ability of NLC/Ce6 to generate ROS under laser stimulation. As previously reported, DPBF may react with ROS to result in UV absorbance quenching at $418 \mathrm{~nm}$ at a UV absorption peak, and the degree of quenching in the environment ROS concentration was positively correlated. This is a convenient way to assess the ability of stimulated ROS generation. As displayed in Fig. 2(b), under laser stimulation, the ROS generation of NLC/Ce6 (Ce6 concentration: $0.1 \mathrm{mg} / \mathrm{mL}$ ) increased significantly, which was reflected in the sharp quenching of the DPBF absorption peak at $418 \mathrm{~nm}$ (79\% at $3 \mathrm{~min})$. These results indicated that NLC/Ce6 in Ce6 retains the generated ROS capacity, which is conducive to play PDT for cancer treatment.

DCFH-DA (2,7-dichlorodi-hydrofluorescein diacetate) can easily penetrate into the inside of the cell, and there is no fluorescence. DCF (2,7-dichlorodi-hydrofluorescein) is produced after intracellular lipase degradation. After ROS oxidation, the fluorescence of DCF is greatly increased compared with unreacted DCF, which provides the possibility to evaluate the intracellular ROS generation spectrum of NLC/Ce6. As displayed in Fig. 3(a), the fluorescence generated in the free $\mathrm{Ce} 6$ group in the absence of stimulation is weak. An increase in fluorescence after exposure to light indicates that laser stimulation is an important factor in the generation of ROS. On the contrary, without laser irradiation, the intracellular fluorescence intensity of the NLC/Ce6 group was stronger than that of the free Ce6 group, which indicated that with the help of DDS, more Ce6 was absorbed into the cells. This is consistent
Figure 1: (a) Size distribution of NLC/ Ce6. (b) Colloidal stability of NLC/Ce6 in PBS (pH 7.4) and mouse plasma at $37^{\circ} \mathrm{C}$ for up to $48 \mathrm{~h}$. Data were shown as mean $\pm \mathrm{SD}(n=3)$.
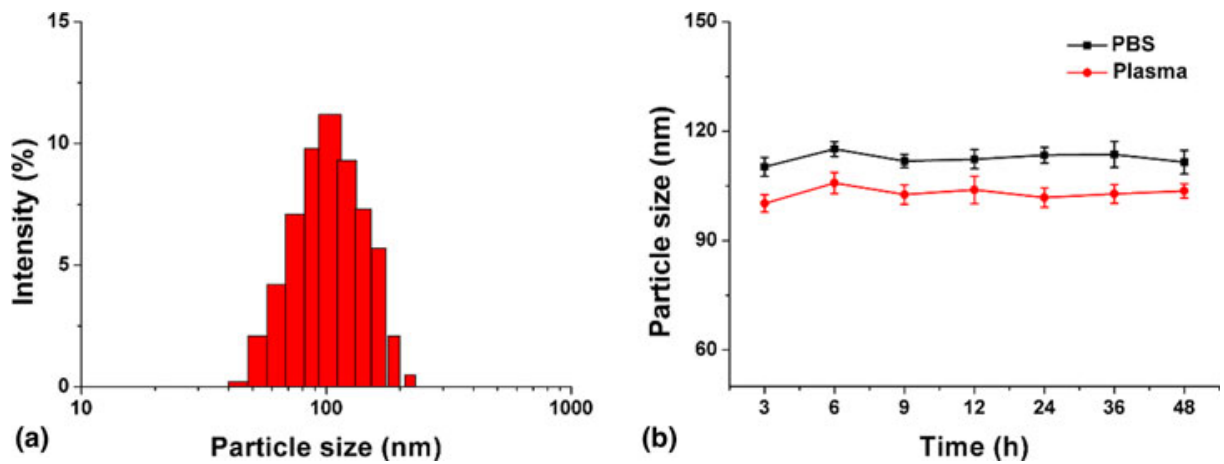


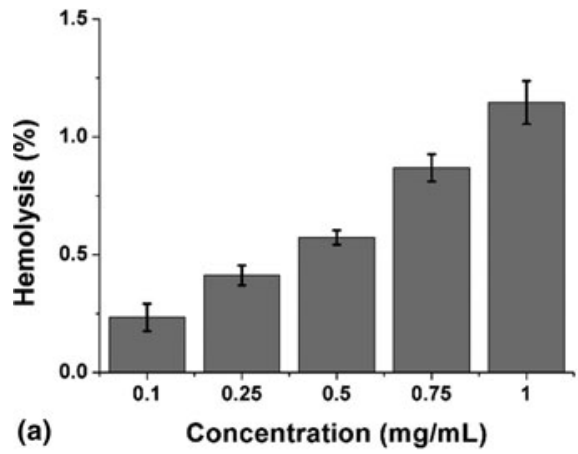

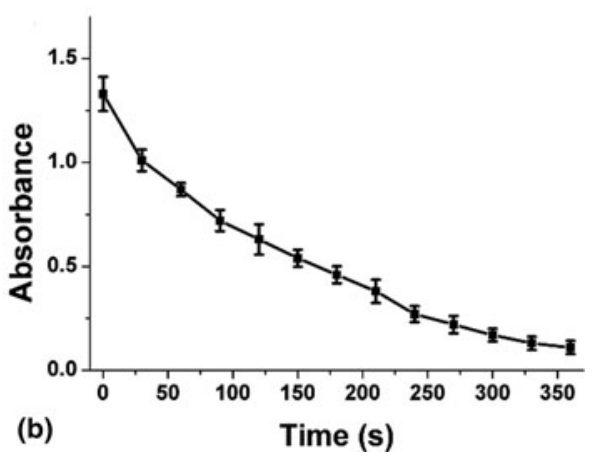

Figure 2: (a) Hemolysis of 2\% RCB incubated with various concentrations of NLC/Ce6. (b) Variation on absorbance of DPBF at $418 \mathrm{~nm}$ in the presence of NLC/Ce6 (Ce6 concentration $0.1 \mathrm{mg} / \mathrm{mL}$ ) after irradiation $\left(1 \mathrm{~W} / \mathrm{cm}^{2}\right)$ for different time intervals. Data were shown as mean $\pm \mathrm{SD}(n=3)$.
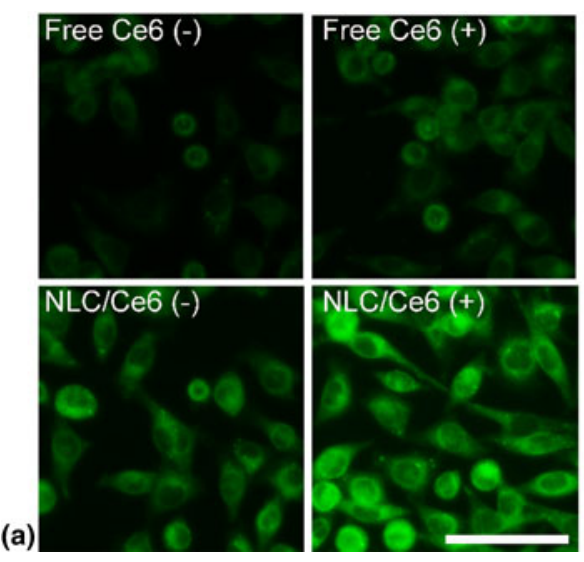

(b)

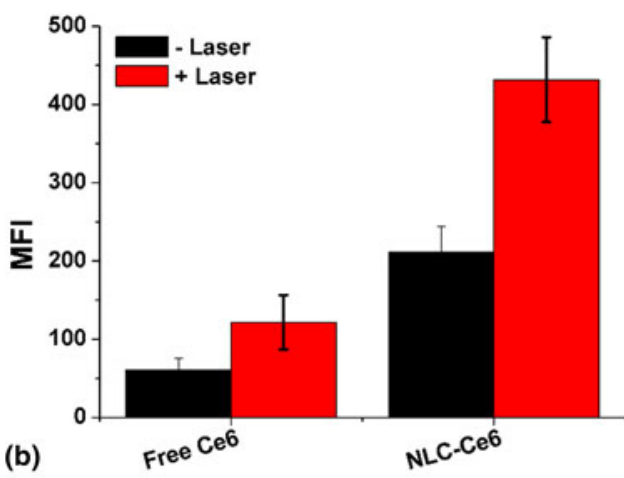

Figure 3: The in vitro ROS generation analysis of NLC/Ce6. (a) The representing CLSM images of cells treated with different formulations with/without laser irradiation. Scale bar: $20 \mu \mathrm{m}$. (b) Main figure illustrated the flow cytometric analysis of MFI (mean fluorescence intensity) in cells treated by different formulations after 5 min of laser irradiation ( $1 \mathrm{~W} /$ $\mathrm{cm}^{2}$ ). Data were shown as mean $\pm \mathrm{SD}(n=3)$. with previous reports that DDS can better deliver drugs into cells through endocytosis. Similarly, after laser irradiation, the fluorescence intensity in the cells treated with NLC/Ce6 was much stronger than the other groups, indicating the best ROS production ability. Using flow cytometry to further quantify the results in Fig. 3(b), it was found that the ROS generation in the NLC/Ce6 group was 3.75 times that of the free Ce6 group after laser irradiation, indicating that the effective delivery effect of NLC/Ce6 on cancer has achieved PDT.

\section{Targetability assessed by cellular uptake in vitro and distribution in vivo}

The cellular uptake of different samples in MFC (mouse gastric carcinoma cell line) cells was studied. Results in Fig. 4(a) showed that in all groups, the intracellular Ce6 signal was positively correlated with incubation time. It should also be noted that free Ce6 shows lower cell accumulation compared with NLC/Ce6. In the experiments of intracellular ROS, it has been proved that DDS can play a beneficial role in increasing the entry of drugs into cells, which is consistent with the observations here. In addition, without FA pretreatment, the NLC/ Ce6 group consistently showed a stronger intracellular Ce6 signal than free Ce6 in all test intervals. Yet, after FA pretreatment, the cellular Ce6 signal of the NLC/Ce6 group dropped sharply, while the free Ce6 hardly changed. These results indicate that FA modification may increase the intrinsic ability of NLC/Ce6 to enter MFC cells through FA-mediated endocytosis.

With the target to double confirm the in vivo tumorhoming ability of NLC/Ce6 on the same cell model, the fluorescence intensity of Ce6 in tumors and other major organs was observed using in vitro imaging. As shown in Fig. 4(b), the NLC of unmodified FA showed poor tumor accumulation while being mainly trapped in major organs, especially the reticuloendothelial system (liver and spleen). Instead, FA-modified NLC/Ce6 tumor tissue in the group Ce6 signal is much higher than in the major organs Ce6 signal, which indicates by FA modifying the surface can improve the FA-unmodified NLC non-targeted distribution.

\section{Anti-cancer effect in vitro}

Thereafter, the anti-cancer effect of drug-loaded NLC/Ce6 was evaluated using free Ce6 as a control. According to Fig. 5(a), it was observed that under the given laser conditions, the PDT effect of all Ce6- containing preparations was positively correlated with the Ce6 concentration. In addition, unlike DDS, free Ce6 showed a poor anti-cancer effect, indicating that DDS can have a beneficial effect on drug delivery $[27,28]$. Most importantly, compared with free Ce6, the benefits of NLC/Ce6 are greatly enhanced at all Ce6 concentrations. Especially when 
Figure 4: (a) Quantitative analysis of intracellular time-dependent uptake of different formulations in MFC cells (pretreated with/without FA). (b) Mean fluorescence intensity of dissected tumors and major organs of mice treated with ICG labeled FA-unmodified/modified NLC/Ce6 at $48 \mathrm{~h}$ post injection. Data were expressed as mean \pm SD $(n=3)$.

Figure 5: (a) Cytotoxicity of free $\mathrm{Ce} 6$ and NLC/Ce6 (with laser irritation) at different $\mathrm{Ce} 6$ concentrations against MFC cells after $48 \mathrm{~h}$ incubation. Inserted images demonstrated the cleaved Caspase-3, cytochrome C, and $\mathrm{BCl}-2$ levels in three groups (Ce6 concentration: $5 \mu \mathrm{g} / \mathrm{mL}$ ). (b) The volume changes of MCTS after different treatments. Data were shown as mean $\pm \mathrm{SD}(n=3)$.
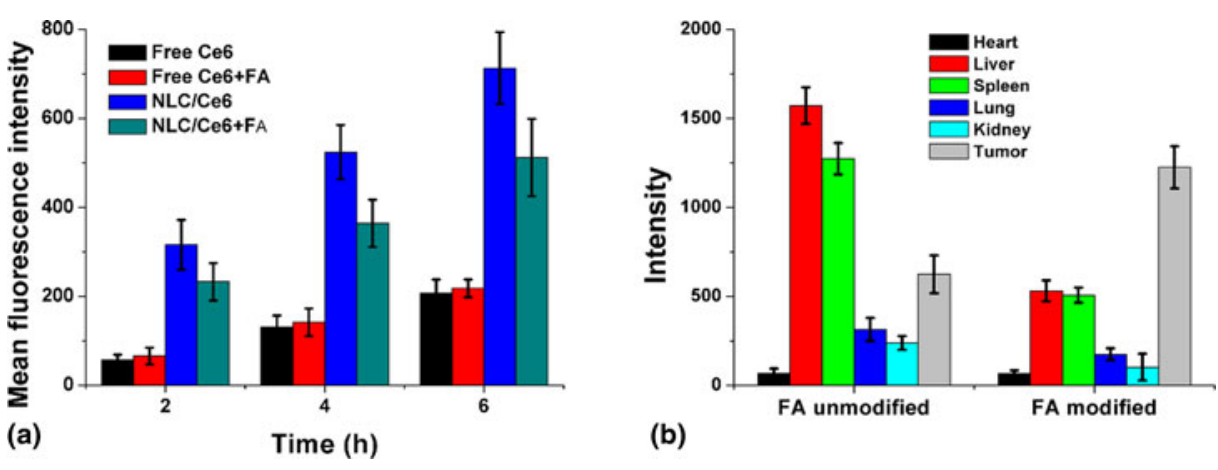

(b)

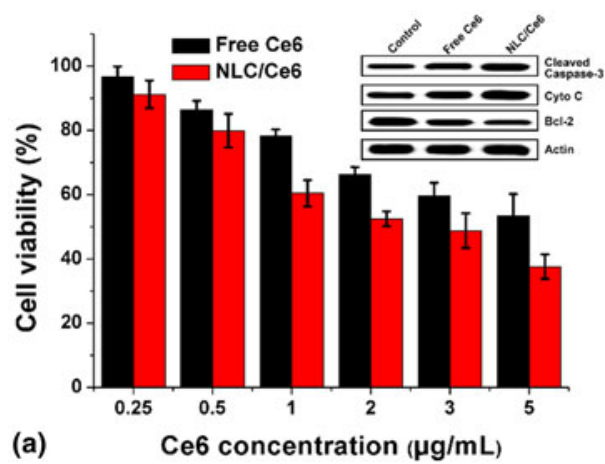

the concentration is $5 \mu \mathrm{g} / \mathrm{mL}$, the cell viability after PDT is less than $40 \%$, which is the best among all tested preparations.

With the aim to study the in vitro anti-cancer effect from another way, western blotting was performed to assess the in vitro variations of the three proteins related to apoptosis. Previous reports have determined that Bcl-2 is an inhibitor of apoptosis and has a beneficial effect on cell survival. Cytochrome $\mathrm{C}$ is another protein that causes apoptosis. The up-regulation of cytochrome $\mathrm{C}$ usually suggests the occurrence of apoptosis, usually followed by the activation of downstream pathways. Eight Caspase-3, as a downstream protein of cytochrome C, usually follows with the beginning of cell apoptosis. The image inserted in Fig. 5(a) shows that PDT can reduce the cellular Bcl-2 level of the cell. The decrease of Bcl-2 is related to the up-regulation of cytochrome $\mathrm{C}$, which further leads to the up-regulation of cleaved Caspase-3. It is worth noting that NLC/Ce6 showed the lowest Bcl-2 level and the highest levels of cytochrome C/Caspase-3 lysis among all groups, which indicates that compared with other groups, cells treated with NLC/Ce6 are experiencing server apoptosis.

Multicellular tumor spheroid (MCTS) composed of fibroblasts and tumor cells are widely used to simulate solid tumors and evaluate the therapeutic effect of DDS. As shown in Fig. 5 (b), the saline group without any treatment showed a continuous increase in the volume of MCTS, which was almost 2.96 times its original size at the end of the experiment, and represented the development of solid tumors. However, the amount of MCTS in all PDT groups was significantly reduced. As noted, in line with the MTT (3-(4,5-dimethylthiazol-2yl)-2,5-diphenyltetrazolium bromide) analysis, NLC/Ce6 showed the best anti-cancer effect with the lowest MCTS volume, thereby reversing the growth rate of MCTS to 0.96 times its original size.

\section{In situ DC vaccination and immune response}

The results of previous studies indicate that PDT destroys the structural integrity of tumors in vivo and mimics the inflammatory process. Therefore, after PDT, the ROS generated in the tumor tissue triggers the recruitment of DCs, and the TAA exposed in the tumor tissues due to apoptosis provides an in situ vaccination to recruit DCs, leading to subsequent immune responses. In addition, the structural integrity of tumor tissue destruction further promotes the spread of DC for adequate vaccination. Therefore, we selected two cytokines (IL-6 and TNF- $\alpha$ ), and their time-dependent plasma concentrations upon PDT were determined by ELISA. As both cytokines are secreted by the activated DC, their level is therefore positively related to DC activation. It was confirmed that TNF- $\alpha$ can directly affect the survival of cancers, whereas IL- 6 in of $\mathrm{CD} 8^{+}$ $\mathrm{T}$ plays a key role in the activation of other killing immune cells. As displayed in Fig. 6, different to the control group, the IL- 6 and TNF- $\alpha$ levels in plasma in the PDT treated group increased after treatment and increased as time extended, 


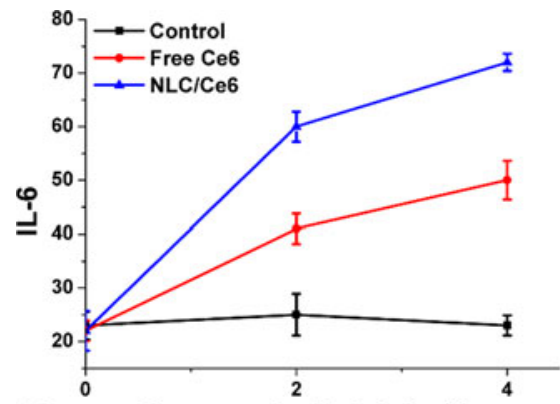

(a)

Days post administration

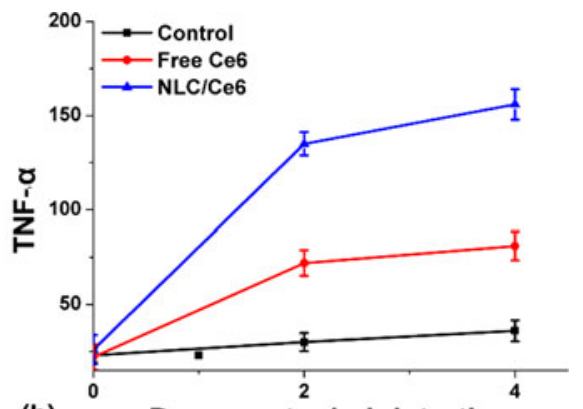

(b)

Days post administration
Figure 6: The levels of (a) IL-6 and (b) TNF- $a$ in sera from mice isolated at 0,2 , and 4 days after different treatments. Data were shown as mean $\pm \operatorname{SD}(n=3)$. revealing a clear activation process. A few days after treatment, it was shown that DC was activated by the in situ vaccine and continued to release target cytokines to induce the following immune response. Moreover, the cytokine level of the NLC/Ce6 group is higher than that of the other groups, which indicated that NLC/Ce6 can be preferably used to be an in situ vaccine for the effective immune response [29].

\section{In vivo anti-cancer research}

With the aim to further verify the role of NLC/Ce6 as an in situ DC cell vaccine in activating the immune response in vivo, we established a mouse model with primary and distant tumors. In brief, randomly assigned mice with number of six were defined as individual test group. Thereafter, using saline as a control, different formulations with the same amount of Ce6 (5 mg/ $\mathrm{kg}$ ) were administered concurrently to the mice. The administration was repeated every 2 days, and PDT was applied to the primary tumor $24 \mathrm{~h}$ after the administration. The tumor volume of the primary and distant tumors was measured on the 3 rd day and repeated every 3 days for 15 days. Experimental results were summarized in Fig. 7. It was observed that the primary tumor and distant tumors were significantly inhibited in NLC/the Ce6 group $\left(223 \mathrm{~m}^{3}\right.$ for primary tumor and $81 \mathrm{~mm}^{3}$ for distant tumor). In contrast, at the end of the test, the tumors in the control group showed steady growth, and the final tumor volumes were $796 \mathrm{~mm}^{3}$ (primary) and $522 \mathrm{~mm}^{3}$ (distant), indicating increased stability and tumor regression after FA modification. All in all, the results strongly indicate that in situ DC vaccination of NLC/Ce6 is of great significance for effective treatment of cancer.

\section{Conclusion}

In our study, an FA-modified NLC was developed as the vehicle to load Ce6 (NLC/Ce6) and finally serve as a potential in situ DC vaccine for effective immunotherapy of gastric cancer. The NLC/Ce6 showed high stability, biocompatibility, and tumor-targetability which can effectively generate ROS upon laser stimulation. The ROS can trigger cell apoptosis to expose TAA and is expected to recruit DC to the tumor tissue, possibly through the inflammation mimicking mechanism. The DCs were activated in situ to release corresponding inflammation (including IL-6 and TNF- $\alpha$ ) and triggering the following immune response cascade. Both in vitro and in vivo experiments suggested the strong capability of this platform to enhance DC vaccination, resulting in effective inhibition of both primary ions and distant growth of gastric cancer, which might be a novel approach for effective therapy of cancer.

\section{Methodology}

\section{Materials}

All chemicals unstated were provided by Sigma-Aldrich (St. Louis, MO). DOPA, oleic acid (OA), and FA-DSPE-PEG were purchased from Ponsure Biotechnology (Shanghai, China).

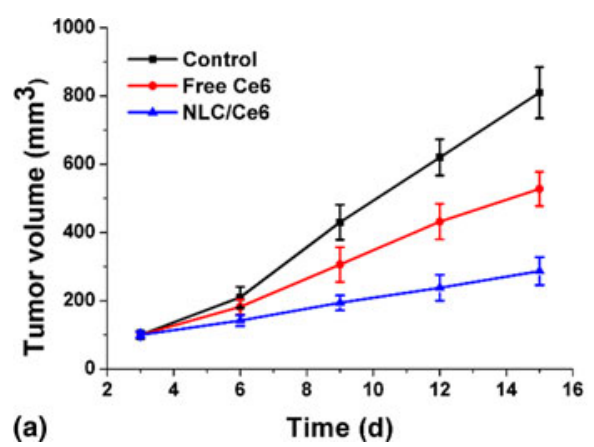

(a)

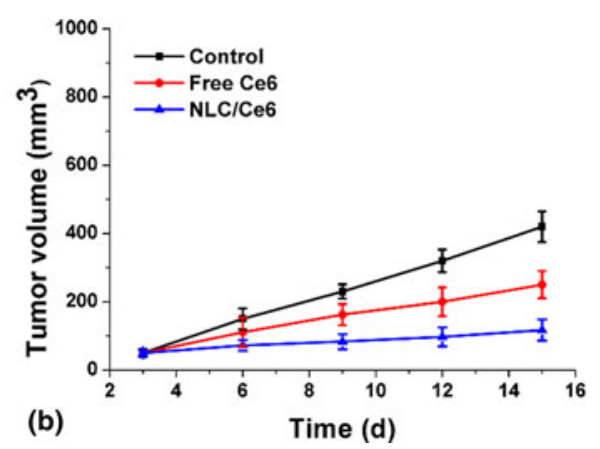

Figure 7: In vivo anti-cancer efficacy of different nano-formulations for MFC tumor-bearing C57BL/6 mice. Tumor volume changes of (a) primary and (b) distant tumors as a function of time were recorded. 


\section{Cell and animal}

MFC (mouse gastric carcinoma cell line) and NIH3T3 (mouse embryonic fibroblast) cell lines were obtained from IBCB center of Shanghai (Shanghai, China). All cell lines were cultured in the standard protocol as reported previously [27]. The MCTSwas established following previous study [19]. Female $\mathrm{Balb} / \mathrm{c}$ nude mice at the age of 6-8 weeks were purchased from Institute of Model Animal Wuhan University (Wuhan, China) and raised in standard conditions, and the MFC tumor xenograft model follows the protocol of previous report [28]. The adopted animal procedures in our experiments were all approved by the Institutional Ethics Committee (IEC) of Guangxi Medical University.

\section{Preparation of $\mathrm{NLC} / \mathrm{Ce} 6$}

OA (2 mg), FA-DSPE-PEG (1 mg), and Ce6 (2 mg) were added into $2 \mathrm{~mL}$ of ethanol and added into a preheated $\left(70^{\circ} \mathrm{C}\right)$ aqueous solution via syringe under gentle agitation ( $500 \mathrm{rpm})$. Then, the solution was centrifuged $(20,000 \times g$ for $10 \mathrm{~min}$, L720R-3, Cence, Changsha, China) to remove unloaded Ce6.

The Ce6 that was content in the precipitation was determined by the ultraviolet spectrophotometer at a wavelength of $405 \mathrm{~nm}$ (Agilent Cary 3500, Agilent, CA) and used to calculate the drug loading content.

\section{Functional test}

The size of NLC/Ce6 in different medium was measured by the size analyzer (ZS90, Malvern, UK). The morphology was shown by transmission electron microscope (Hitachi-2100, Hitachi, Japan) [30].

The hemolysis was assessed by standard protocol reported in previous research [31]. The western blot assay was conducted according to standard protocol using antibodies from Cell Signaling Technology (CST, Boston, USA) and imaged by (Chemstudio touch, Analytik Jena AG, Germany). The IL-6 and TNF- $\alpha$ levels were assayed using an ELISA kit (Thermo Fisher, Massachusetts, USA) as instructed.

\section{In vitro anti-cancer assay}

NLC $(5-100 \mu \mathrm{g} / \mathrm{mL})$ or NLC/Ce6 (Ce6 concentration, 0.25$5 \mu \mathrm{g} / \mathrm{mL}, 680 \mathrm{~nm}, 400 \mathrm{~mW} / \mathrm{cm}^{2}$ for $10 \mathrm{~min}$ ) were incubated with MFC cells $(48 \mathrm{~h})$. Afterwards, MTT assay was applied reported previously to study cell viability [32]. In addition, the MCTS was incubated with different formulations at a Ce 6 concentration of $5 \mu \mathrm{g} / \mathrm{mL}$ for $24 \mathrm{~h}$ and then subjected to laser irradiation. The MCTS volume was monitored for 5 days.

\section{Cellular uptake and ROS}

MFC cells pretreated with or without FA $(2 \mathrm{~h})$ were cultured with free $\mathrm{Ce} 6$ and NLC/Ce6. At different time intervals, cells were harvested and subjected to flow cytometer (DxFLEX, Beckman Coulter, Miami, FL) analyzation.

The intracellular ROS levels were evaluated according to previous report. In brief, cells were treated with fresh serumfree medium containing DCFH-DA $(25 \mathrm{mM})$ and different samples for $4 \mathrm{~h}$ in the dark for $30 \mathrm{~min}$. Finally, cells were irradiated with/without laser irradiation $\left(1 \mathrm{~W} / \mathrm{cm}^{2}, 5 \mathrm{~min}\right)$ and then imaged by confocal laser scanning scope (CLSM; FV3000, Olympus, Tokyo, Japan).

\section{In vivo distribution}

MFC tumor-bearing mice with only primary tumor were intravenously injected with FA-unmodified/modified NLC/Ce6. At $48 \mathrm{~h}$ post injection, the tumor tissues and other major organs were collected to visualize their Ce6 distribution using the in vivo imaging system (ZEWTON 7.0, Vilber, France).

\section{In vivo anti-cancer assay}

Mice randomly divided $(n=6)$ were administered with saline (as control), free Ce6, and NLC/Ce6. The primary tumor was irradiated by $680 \mathrm{~nm}$ laser at $100 \mathrm{~mW} / \mathrm{cm}^{2}$ for $20 \mathrm{~min}$. The administration was performed every 2 days for two times at a Ce6 concentration of $5 \mathrm{mg} / \mathrm{kg}$, while the irradiation was performed $24 \mathrm{~h}$ post drug administration [33]. The tumor volumes of all subjects were measured using caliper at day 3 every 3 days for 15 days.

\section{Acknowledgments}

We acknowledge the financial support from the National Natural Science Foundation of China (No. 81660134) and the Natural Science Foundation of Guangxi Province (Nos. 2016GXNSFAA380162 and 2017GXNSFAA198051).

\section{Reference}

1. M. Neek, T.I. Kim, and S.-W. Wang: Protein-based nanoparticles in cancer vaccine development. Nanomedicine-Nanotechnol. 15, 164 (2019).

2. M. Saxena and N. Bhardwaj: Re-emergence of dendritic cell vaccines for cancer treatment. Trends Cancer 4, 119 (2018).

3. B. Mastelic-Gavillet, K. Balint, C. Boudousquie, P.O. Gannon, and L.E. Kandalaft: Personalized dendritic cell vaccines-Recent breakthroughs and encouraging clinical results. Front. Immunol. 10, 766 (2019). 
4. A. Mougel, M. Terme, and C. Tanchot: Therapeutic cancer vaccine and combinations with antiangiogenic therapies and immune checkpoint blockade. Front. Immunol. 10, 467 (2019).

5. N. Dobrovolskienė, V. Pašukonienė, A. Darinskas, J.A. Kraśko,

K. Žilionytė, A. Mlynska, Ž Gudlevičienè,

E. Mišeikytè-Kaubrienė, V. Schijns, and W. Lubitz: Tumor lysate-loaded Bacterial Ghosts as a tool for optimized production of therapeutic dendritic cell-based cancer vaccines. Vaccine $\mathbf{3 6}$ 4171 (2018).

6. N.P. Restifo, M.E. Dudley, and S.A. Rosenberg: Adoptive immunotherapy for cancer: Harnessing the T cell response. Nat. Rev. Immunol. 12, 269 (2012).

7. K. Palucka, and J. Banchereau: Cancer immunotherapy via dendritic cells. Nat. Rev. Cancer 12, 265 (2012).

8. C. Wang, F. Yu, X. Liu, S. Chen, R. Wu, R. Zhao, F. Hu, and H. Yuan: Cancer-specific therapy by artificial modulation of intracellular calcium concentration. Adv. Healthcare Mater. 8, 1900501 (2019).

9. X. Yang, T. Yu, Y. Zeng, K. Lian, X. Zhou, S. Li, G. Qiu, X. Jin, H. Yuan, and F. Hu: Tumor-draining lymph node targeting chitosan micelles as antigen-capturing adjuvants for personalized immunotherapy. Carbohydr. Polym. 240, 116270 (2020).

10. C. Wang, S. Chen, L. Bao, X. Liu, F. Hu, and H. Yuan: Size-controlled preparation and behavior study of phospholipidcalcium carbonate hybrid nanoparticles. Int. J. Nanomed. 15, 4049 (2020).

11. M. Li, Y. Liu, J. Chen, T. Liu, Z. Gu, J. Zhang, X. Gu, G. Teng, F. Yang, and N. Gu: Platelet bio-nanobubbles as microvascular recanalization nanoformulation for acute ischemic stroke lesion theranostics. Theranostics 8, 4870 (2018).

12. R. Wen, A.C. Umeano, Y. Kou, J. Xu, and A.A. Farooqi: Nanoparticle systems for cancer vaccine. Nanomedicine 14, 627 (2019).

13. R.R. Meka, S. Mukherjee, C.R. Patra, and A. Chaudhuri: Shikimoyl-ligand decorated gold nanoparticles for use in ex vivo engineered dendritic cell based DNA vaccination. Nanoscale 11, 7931 (2019).

14. R.P.M. Dings, M. Cannon, and K.B. Vang: Design of gold nanoparticles in dendritic cell-based vaccines. Part. Part. Syst. Charact. 35, 1800109 (2018).

15. X. Zhao, D. Tang, T. Yang, and C. Wang: Facile preparation of biocompatible nanostructured lipid carrier with ultra-small size as a tumor-penetration delivery system. Colloids Surf., B 170, 355 (2018).

16. C. Tapeinos, A. Marino, M. Battaglini, S. Migliorin, R. Brescia, A. Scarpellini, C. De Julián Fernández, M. Prato, F. Drago, and G. Ciofani: Stimuli-responsive lipid-based magnetic nanovectors increase apoptosis in glioblastoma cells through synergic intracellular hyperthermia and chemotherapy. Nanoscale 11, 72 (2019).
17. P. Tambe, P. Kumar, K.M. Paknikar, and V. Gajbhiye:

Decapeptide functionalized targeted mesoporous silica nanoparticles with doxorubicin exhibit enhanced apoptotic effect in breast and prostate cancer cells. Int. J. Nanomed. 13, 7669 (2018).

18. X. Sun, J. Sun, J. Lv, B. Dong, M. Liu, J. Liu, L. Sun, G. Zhang, L. Zhang, and G. Huang: Ce6-C6-TPZ co-loaded albumin nanoparticles for synergistic combined PDT-chemotherapy of cancer. J. Mater. Chem. B 7, 5797 (2019).

19. G. Jin, R. He, Q. Liu, M. Lin, Y. Dong, K. Li, B.Z. Tang, B. Liu, and F. Xu: Near-infrared light-regulated cancer theranostic nanoplatform based on aggregation-induced emission luminogen encapsulated upconversion nanoparticles. Theranostics 9, 246 (2019).

20. J. Yang, Y. Teng, Y. Fu, and C. Zhang: Chlorins e6 loaded silica nanoparticles coated with gastric cancer cell membrane for tumor specific photodynamic therapy of gastric cancer. Int. J. Nanomed. 14, 5061 (2019).

21. C. He, X. Duan, N. Guo, C. Chan, C. Poon, R.R. Weichselbaum, and W. Lin: Core-shell nanoscale coordination polymers combine chemotherapy and photodynamic therapy to potentiate checkpoint blockade cancer immunotherapy. Nat. Commun. 7, 1 (2016).

22. S. Ma, J. Zhou, Y. Zhang, B. Yang, Y. He, C. Tian, X. Xu, and Z.J.A.A.M. Gu: An oxygen self-sufficient fluorinated nanoplatform for relieved tumor hypoxia and enhanced photodynamic therapy of cancers. ACS Appl. Mater. Interfaces 11(8), 7731-7742.

23. H. He, R. Zhu, W. Sun, K. Cai, Y. Chen, and L. Yin: Selective cancer treatment via photodynamic sensitization of hypoxia-responsive drug delivery. Nanoscale 10, 2856 (2018).

24. R. Hu, H. Zheng, J. Cao, Z. Davoudi, and Q. Wang: Synthesis and in vitro characterization of carboxymethyl chitosan-CBAdoxorubicin conjugate nanoparticles as $\mathrm{pH}$-sensitive drug delivery systems. J. Biomed. Nanotechnol. 13, 1097 (2017).

25. F. Gao, J.M. Zhang, C.M. Fu, X.M. Xie, F. Peng, J.S. You, H.L. Tang, Z.Y. Wang, P. Li, and J.P. Chen: iRGD-modified lipid-polymer hybrid nanoparticles loaded with isoliquiritigenin to enhance anti-breast cancer effect and tumor-targeting ability. Int. J. Nanomed. 12, 4147 (2017).

26. L. Brillault, P.V. Jutras, N. Dashti, E.C. Thuenemann, G. Morgan, G.P. Lomonossoff, M.J. Landsberg, and F. Sainsbury: Engineering recombinant virus-like nanoparticles from plants for cellular delivery. ACS Nano. 11, 3476 (2017).

27. L.M. Negi, S. Talegaonkar, M. Jaggi, and A.K. Verma: Hyaluronated imatinib liposomes with hybrid approach to target CD44 and P-gp overexpressing MDR cancer: an in-vitro, in-vivo and mechanistic investigation. J. Drug Target. 27, 183 (2019).

28. C. Wang, Z. Wang, X. Zhao, F. Yu, Y. Quan, Y. Cheng, and H. Yuan: DOX loaded aggregation-induced emission active polymeric nanoparticles as a fluorescence resonance energy transfer traceable drug delivery system for self-indicating cancer therapy. Acta Biomater. 85, 218 (2019). 
29. J. Ni, Y. Sun, J. Song, Y. Zhao, Q. Gao, and X. Li: Artificial cell-mediated photodynamic therapy enhanced anticancer efficacy through combination of tumor disruption and immune response stimulation. ACS Omega 4, 12727 (2019).

30. X. Ding, X. Xu, Y. Zhao, L. Zhang, Y. Yu, F. Huang, D. Yin, and H. Huang: Tumor targeted nanostructured lipid carrier co-delivering paclitaxel and indocyanine green for laser triggered synergetic therapy of cancer. RSC Adv. 7, 35086 (2017).

31. C. Wang, M. Han, X. Liu, S. Chen, F. Hu, J. Sun, and H. Yuan: Mitoxantrone-preloaded water-responsive phospholipid-amorphous calcium carbonate hybrid nanoparticles for targeted and effective cancer therapy. Int. J. Nanomed. 14, 1503 (2019).

32. X. Zheng, F. Zhang, Y. Zhao, J. Zhang, J. Dawulieti, Y. Pan, L. Cui, M. Sun, D. Shao, and M. Li: Self-assembled dual fluorescence nanoparticles for CD44-targeted delivery of anti-miR-27a in liver cancer theranostics. Theranostics 8, 3808 (2018).

33. X. Zhao, Y. Liu, Y. Yu, Q. Huang, W. Ji, J. Li, and Y. Zhao: Hierarchically porous composite microparticles from microfluidics for controllable drug delivery. Nanoscale 10, 12595 (2018). 